\title{
Funerary Imprecations in the Balcan Provinces ${ }^{1}$
}

\author{
Lyuba Radulova
}

Rita Sassu

\section{Doi:10.5901/ajis.2015.v4n1p469}

\section{Abstract}

The paper aims at collecting and analysing the inscriptions from the Balcan provinces which contain imprecations addressed to potential grave violators. One surprising result of the analysis is the fact that the inscriptions with funerary imprecations are quite an insignificant part of the total number of the funerary inscriptions found in the area. Taking into consideration the fact that the topoi contained in the attested imprecations demonstrate a clear microasiatic influence, il seems quite likely that the investigated funerary imprecations should be a phenomenon coming from the East. As far as chronology is concerned, it is possible to note a strong disproportion in the distribution of the imprecations, their peak being in the period between the fourth and the sixth century. This peak coincides with the appearance of a new type of funerary imprecation in which the Christian God is invoked to protect the tomb. On the basis of these observations the paper offers the more general conclusion that funerary imprecations in the Balcan provinces can be perceived as reflecting the ethnic and religious changes in the area during the period of the late Antiquity.

Keywords: funerary imprecations; tomb violation; Balcan provinces; late Antiquity.

\section{Introduzione. La tomba come ultima dimora del defunto}

La morte rappresenta un momento costitutivo della società umana, configurandosi presso diverse civiltà come una delle basi formative per lo sviluppo della religione, della visione cosmogonica e della cultura sociale. La tomba, sede perpetua del defunto, costituisce un oggetto di studio complesso, il cui interesse non appare limitato alla ricerca inerente alla struttura fisica del monumento e al relativo materiale archeologico ed epigrafico, ma si estende invece a diverse sfere di indagine, connesse alla restituzione delle credenze, delle strutture ideologiche e religiose, ma anche politiche, della società che ha prodotto la tomba stessa. Quindi il sepolcro, luogo ove è operata una sintesi delle concezioni fondanti di una società, rappresenta, se affrontato come sistema complesso, uno strumento d'eccellenza per la restituzione dei cambiamenti di mentalità e di percezione del mondo verificatisi durante le diverse epoche storiche.

La preoccupazione per la tutela della tomba risponde a molteplici esigenze, avvertite tanto dalle culture passate quanto da quelle presenti, e diversi sono i fattori che concorrono a determinare la necessità di elaborare misure efficienti volte a prevenirne l'eventuale violazione.

In prima istanza, la tomba si configura sovente come uno strumento funzionale a preservare la memoria del morto, non permettendo che cada nell'oblio, ma continui invece ad essere eternata, non solo per mezzo del monumento funebre, ma anche attraverso l'impiego di testi o immagini. In questo modo, la tomba può, in alcuni casi, divenire una sorta di spazio pubblico dove è illustrata e sintetizzata la vita (o un momento saliente) del defunto, talvolta proposta come modello ai vivi, secondo una logica rappresentativa che può trovare espressione nel linguaggio figurativo e filologico.

In molti casi, la preoccupazione per la protezione della sepoltura appare correlata ad una credenza positiva nella vita oltre la morte, che può anche concepire l'esistenza ultraterrena del defunto come direttamente connessa allo stato e all'integrità del corpo dell'individuo morto, quando non addirittura ritenere la tomba il luogo stesso della vita post-mortem. Tale situazione fa sì che in diversi contesti sepolcrali sia possibile rinvenire una scelta di oggetti reputati importanti per il defunto e pertanto con lui seppelliti. Proprio la presenza di oggetti in ambito sepolcrale costituisce una delle più frequenti motivazioni delle azioni di depredazione del luogo di sepoltura, che rappresentano, unitamente all'inserimento di ulteriori defunti in un sito già occupato, le ragioni più frequenti di violazione del sepolcro.

In diverse civiltà, inoltre, sussiste la convinzione che il defunto, qualora non sepolto, possa riscontrare una serie di

${ }^{1}$ Si ringraziano per la revisione del testo e i suggerimenti: la prof.ssa M.L. Caldelli, il prof. L. Caliò, il Dott. A. Filippini, il prof. E. Lippolis, il prof. M. Mazza. 
problemi, che possono addirittura procurargli l'impossibilità di pervenire alla quiete oltre la morte, facendone infine un elemento pericoloso per gli stessi viventi.

La tomba non rappresenta, difatti, esclusivamente uno spazio incentrato sul morto, legato al suo ricordo e alla sua serenità post-mortem, ma essa, congiuntamente ai riti funerari, mira altresì a garantire la tranquillità dei vivi, segnando una linea di demarcazione tra questi e i defunti e rendendo, per così dire, 'inoffensivi' i secondi. La tomba, luogo in cui si accentrano elementi commemorativi, cultuali e culturali, diviene così luogo dell'interazione fra i vivi e i morti, beneficiari di preghiere, rituali e offerte.

Affinché i vivi siano informati della presenza di tale locus religiosus, la tomba è spesso monumentalizzata, al fine di segnare topograficamente il luogo dei morti, dove i vivi possono entrare in contatto con questi.

Presso molteplici civiltà, infine, il sepolcro è concepito quale ultima dimora, segnata da caratteri di eternità e perpetuità, nonché luogo del refrigerium, del riposo postumo, come spesso espresso nei testi funerari.

Questi sono solo alcuni dei fattori da cui deriva l'avvertita esigenza, da parte di molte civiltà, non solo di creare la tomba - si ricordi, tuttavia che in alcune culture la sepoltura formale può non essere destinata a tutti, ma essere invece un 'privilegio' riservato a particolari categorie - ma anche di apporvi testi scritti, volti sia, come accennato, a tramandare la memoria del defunto, sia a preservare la sepoltura stessa attraverso la creazione di una barriera di difesa quanto più efficace possibile, che può avere le sue fondamenta tanto su basi giuridiche che religiose. I testi, infatti, possono dissuadere il potenziale reo ricordando le pene legali scaturenti dall'atto di violazione, ma anche formulare vere e proprie maledizioni: l'epigrafe può far riferimento sia alla legge umana e alle sanzioni da essa prevista, sia alla legge religiosa dominata da referenti ultraterreni, anche divini, incaricati di punire l'atto di empietà; anche laddove non è menzionato alcun ente soprannaturale incaricato del castigo del violatore, la credenza nel potere della parola scritta, espressa tramite la maledizione, ne fa apparire indubbia l'efficacia.

Il campo d'indagine sui mezzi religiosi per assicurare l'inviolabilità della sepoltura, in larga misura coincidente con il settore di studio delle maledizioni, è stato approfondito, nella prima metà del XX secolo, da A. Parrot nel noto Maledictions et Violations de Tombes, ${ }^{2}$ ove veniva preso in esame l'Oriente antico (a partire dal mondo assirobabilonese a quello sirio-fenicio, all'oriente greco e romano), facendo emergere la sostanziale difficoltà, in un ambito di ricerca così eterogeneo, di tracciare una chiara linea evolutiva di tale tactique défensive. ${ }^{3}$

II presente contributo affronta un'area geograficamente più ristretta rispetto a quella indagata dal Parrot, concentrandosi sull'area balcanica con specifico riferimento alla documentazione rinvenuta nelle province di Dacia, Dalmazia, Illirico, Moesia Superiore e Inferiore, Tracia e Macedonia e prendendo in considerazione sia le iscrizioni in lingua greca che quelle latine. Sulla base dei dati raccolti e di seguito esposti, s'intende mostrare come sia oggi, forse, possibile individuare un paradigma di sviluppo diacronico nell'affermazione e diffusione delle maledizioni sepolcrali nello spazio fatto oggetto d'indagine.

\section{Premessa allo studio le maledizioni sepolcrali nelle province balcaniche}

Prima di affrontare la problematica relativa alle maledizioni sepolcrali rinvenute nelle province balcaniche dell'Impero Romano, appare opportuno, al fine di poter meglio inquadrare il fenomeno nel suo sviluppo diacronico, accennare succintamente all'origine stessa della maledizione sepolcrale, le cui prime espressioni risultano registrabili nell'Antico Oriente.

Nonostante la formula della maledizione sia già nota al mondo assiro-babilonese, ${ }^{4} \mathrm{i}$ primi testi funzionali alla salvaguardia della tomba non si presentano come maledizioni esplicite, quanto piuttosto come inviti a non toccare il luogo di sepoltura.

I più antichi esempi di maledizioni sepolcrali sono riscontrabili invece nel mondo sirio-fenicio. Tra le attestazioni maggiormente esemplificative, può essere citata la maledizione iscritta sul sarcofago della tomba 5 della necropoli reale di Byblos, che recita: «Itoba'al, figlio di Ahiram, re di Gebal, ha fatto questo sarcofago per Ahiram suo padre, come sua dimora per l'eternità. Se un re tra i re, un governatore fra i governatori, predispone un accampamento per combattere

\footnotetext{
2 Parrot 1939.

3 Per una bibliografia essenziale sul tema, si vd. Libitina 2004; Cuq 1932: 109-126; Huschke 1874; Latte 1920; Lecrivain 1904: $2014-$ 2020; Mommsen 1907: 198-214; Toynbee 1996; De Visscher 1953: 175-183; Giorgi 1910; Parrot 1939; Robert 1978: 241-189; Robert 1960; Strubbe 1991; Strubbe 1997; Tosi 1993, 189-240; Valois 1914: 250-271.

${ }^{4}$ Cfr. la maledizione di Eannadu re di Lagash rivolta agli abitanti di Gish-hu nel caso in cui non rispettino il patto stipulato, o, successivamente, le molteplici minacce rivolte a coloro che intendano alterare iscrizioni o monumenti pubblici.
} 
Gebal e scoperchia questo sarcofago, sia frantumato lo scettro del suo potere, crolli il trono della sua monarchia e la pace regni su Gebal. Quanto a lui, la sua discendenza muoia di spada». ${ }^{5}$ La maledizione che si abbatte sulla discendenza rappresenta un elemento che troverà largo riscontro in tutta l'antichità orientale. ${ }^{6}$

In Egitto, ove si assiste, in ambito religioso, ad una vera a propria centralizzazione della morte, il rito di passaggio per eccellenza corrisponde alla transizione dalla vita terrena a quella ultraterrena. La natura stessa della religione egizia e la credenza nella vita post-mortem fa sì che più mezzi vengano adottati per sottrarre la tomba a qualsiasi tipo di violazione, in primis la depredazione. Tra i vari contenuti delle maledizioni indirizzate al potenziale violatore, ampiamente studiate da $\mathrm{H}$. Sottas nel celebre La préservation de la propriéte funérarie dans l'ancienne Egypte, ${ }^{7}$ figurano frequentemente la minaccia di comparire davanti al Grande Dio (Osiris); l'augurio di «essere assalito in mare dal coccodrillo e dal serpente in terra»; in molti casi le maledizioni non riguardano direttamente la vita materiale, ma investono invece la sfera religiosa, augurando al colpevole di violatio di non ricevere cerimonie funebri né di essere destinatario di alcuna libazione e che le offerte dedicategli dai vivi possano non giovargli: la maledizione agisce quindi anche dopo la morte del reo, influenzandone la vita postuma. Ancora in ambito egizio, nel Nuovo Regno si afferma una tipologia di maledizione destinata a trovare seguito in molteplici civiltà posteriori: il violatore del sepolcro sarà escluso dalle funzioni pubbliche, morirà anzitempo e sarà privato della sepoltura. Nel mondo egizio, pertanto, la maledizione sepolcrale risulta largamente attestata, in quanto ad essa viene attribuita un'efficacia indiscutibile. Basata sulla convinzione dell'esistenza di forze ultra-terrene in grado di agire per punire i trasgressori, la maledizione funeraria egizia colpisce tanto il profanatore della tomba quanto colui che vi si accosta in stato di impurità ed è spesso accompagnata dall'invito, rivolto al passante, di compiere azioni rituali propizie al defunto (aspersioni, offerte, etc.).

L'analisi del mondo greco richiederebbe in realtà la trattazione della complessa tematica dei rapporti tra Oriente $\mathrm{e}$ Occidente, che porterebbe tuttavia lontano dall'intento del presente contributo, incentrato in realtà su una specifica sezione del mondo greco-romano. Ci si limita pertanto a sottolineare come in Grecia la maledizione sepolcrale sia complessivamente abbastanza rara ${ }^{8}$ e ancora meno attestata sia la menzione di pene pecuniarie, prevalendo invece la prassi di porre la tomba sotto la tutela di divinità infere - in prevalenza Plutone, Demetra, Kore, le Erinni, Giove ctonio. Tra formule maggiormente attestate figurano l'augurio che «il mare non sia navigabile» nonché l'eliminazione della posterità.

Nel tentativo di comprendere la ragione della scarsezza di maledizioni funerarie in ambito greco è stata addotta come causa l'assenza di interesse reale, nella religione greca, per gli aspetti materiali della vita oltre la morte, vista perlopiù come pallido riflesso della vita terrena, laddove prevale invece l'importanza attribuita alla sepoltura, ai riti funerari e alla memoria. ${ }^{9}$

Nonostante la situazione descritta dalle epigrafi del mondo greco non sembri indicare un'eccesiva preoccupazione

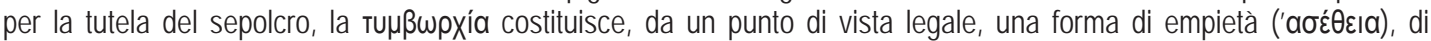

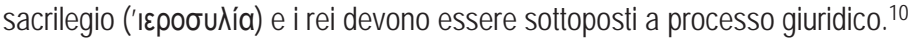

Per quanto concerne il mondo romano, senza ripercorrere in questa sede i complessi sviluppi del diritto in materia sepolcrale, ${ }^{11}$ è sufficiente ricordare come lo iustum sepulcrum rappresenti la condizione imprescindibile per la quiete del morto e la violazione della tomba sia quindi contrastata giuridicamente, principalmente dall'editto pretorio - actio sepulchri violati - che prevede che il reo venga punito con una pena finanziaria - multa sepulchri - riscossa dai possessori della tomba danneggiata in un primo tempo e, successivamente, da una cassa pubblica oppure sacra (e infine dalla Chiesa).

L'actio sepulchri violati sarebbe stata introdotta a seguito dell'inapplicabilità della pena capitale prevista in una fase anteriore, in cui il colpevole, secondo il diritto pontificale, è punito con la devotio o consacrazione agli Dei Inferi, essendo la profanazione del sepolcro intesa come un'offesa agli Dei Mani e pertanto colpa inespiabile. La devotio, mostratasi inefficace in quanto eccessivamente severa e conseguentemente raramente applicata, è sostituita quindi dall'introduzione dell'editto del pretore.

\footnotetext{
${ }^{5}$ Circa 1000 a.C.

${ }^{6}$ Cfr., tra le più celebri, la maledizione proposta da Melishihu, re Kassita: «... che gli Dei cancellino il suo nome (del violatore della tomba), la sua semenza e la sua posterità .... XII secolo a.C.

7 Sottas 1913.

${ }^{8}$ Parrot 1939: 150; Strubbe 1991: 40.

${ }^{9}$ Strubbe 1991: 33-59.

10 Cumont 1930: 263. Cfr. CIG 2834, 2850, 4253, 4307.

11 Per una panoramica complessiva sul diritto sepolcrale romano si vd. De Visscher 1963; Lazzarini 1991.
} 
La nota iscrizione di Nazaret, ${ }^{12}$ variamente datata in un arco temprale compreso tra il 30 a.C. e il 198 d.C., indica un nuovo inasprimento dei mezzi giuridici impiegati per limitare la violazione delle tombe, testimoniando la reintroduzione della pena di morte - tuttavia, non vi sono dati per stabilire che tale sanzione fosse applicata anche al di fuori del territorio palestinese.

Nel II secolo d.C., la necessità di limitare il numero, evidentemente crescente, di tombe violate, comporta un rafforzamento delle pene previste, stabilite in base alle circostanze del reato e alla classe di appartenenza del trasgressore: si prevedono così deportazioni per gli honestiores, lavori pubblici per gli humiliores, pene capitali in caso di violazione 'a mano armata'.

Le pene giuridiche conoscono un'ulteriore inasprimento a partire dal III secolo d.C. Le sanzioni, ancora più severe che nel secolo precedente, prevedono deportazioni, lavori forzati, morte, multe (Costanzo, dopo aver abolito la pena capitale, la ristabilisce nel 356 d.C.). Vari castighi sono destinati a coloro che danneggiano la sepoltura spostando salme, introducendo nuovi defunti, sovrapponendo cadaveri, inserendo nuovi morti in sepolcri già utilizzati. Le pene, in questi casi, risultano essere per lo più di natura finanziaria, corrispondendo a somme da versare allo Stato, ai tesori dei templi, al collegio delle vestali, etc. Parrot registra un'intensificazione, nelle iscrizioni, della menzione delle sanzioni giuridiche in luogo delle maledizioni e attribuisce il fenomeno al venir meno della potenza vendicatrice degli Dei, dapprima mai messa in discussione, e dallo 'scetticismo dilagante', che spinge verso l'individuazione e l'attuazione di pene 'più concrete'.

Tuttavia, dall'esame dei dati di seguito esposti sembrerebbe emergere un quadro sensibilmente differente da quello tracciato dal Parrot per quanto concerne l'età tardo-antica, almeno relativamente alle province analizzate in questa sede. L'area, per nulla dominata dal summenzionato scetticismo dilagante, conosce, come del resto anche altre zone dell'Impero, il diffondersi della religione cristiana, che recupera, come più avanti illustrato, culture più antiche che in essa confluiscono, comportando parallelamente un aumento della preoccupazione per la salvaguardia della tomba dei defunti - destinati ad una vita eterna oltre la morte -, come si evince dalle epigrafi funerarie di seguito analizzate, ove la menzione delle pene fiscali si accompagna di norma con formule di maledizione intrinsecamente legate alla nuova fede, che da un lato raccolgono elementi della tradizione più antica appropriandosene, dall'altro riflettono la nuova mentalità del periodo identificato nella Tarda Antichità. ${ }^{13}$

Le pene fiscali sono in quest'epoca destinate tanto al fisco, ${ }^{14}$ quanto alla Chiesa. ${ }^{15}$ Un altro fattore di rilievo è infatti rappresentato dalla presa di possesso dell'amministrazione cimiteriale da parte della Chiesa romana.

\section{Le maledizioni nelle province balcaniche}

Ai fini del presente lavoro è seguita la definizione di maledizione proposta nel 1997 da J.H.M. Strubbe, ${ }^{16}$ il quale, nel tentativo di tratteggiare una chiara linea di demarcazione tra le defixiones e le maledizioni sepolcrali, circoscrive il termine imprecations alle sole maledizioni, iscritte in maniera chiara e pubblica sul monumento funerario dal proprietario della tomba, il quale non nasconde la sua identità, allo scopo di rendere noti a ogni potenziale violatore del sepolcro i mali e le pene da cui sarà afflitto. ${ }^{17}$

All'interno delle maledizioni sepolcrali, inoltre, si tiene conto della distinzione, anch'essa operata da Strubbe, ${ }^{18}$ tra 'imprecazioni specifiche'19 e 'imprecazioni non specifiche'. ${ }^{20}$ Nel primo gruppo rientrano le maledizioni che contengono una descrizione concreta del castigo che colpirà l'eventuale violatore. In questo caso la scelta della punizione è opera del

\footnotetext{
12 Editio princeps in Cumont 1930: 241-266. Per una bibliografia critica sull'epigrafe, si vd. Tsalampouni 2001: 70-120.

13 Vd., a titolo esemplificativo, ILCV 3845: male pereat insepultus jaceat non resurgat cum Juda partem habeat si quis hunc sepulcrum violaverit o CIL XI 322 = ILCV 3850: si quis sepulchrum violaverit partem habeat cum Jude traditorem et in die judicii non resurgat.

${ }^{14} \mathrm{Vd}$., ad es., l'iscrizione di $\mathrm{V}$ secolo rinvenuta ad Ancona: si quis violentus voluerit esse et contra leges temtaverit det fisci viribus auri libra una.

15 ILS 8252, Salona, Dalmazia V d.C.: quiscumque extraneus voluerit alterum corpus ponere det ecclesiae catholicae Salonitanae auri tres.

16 Strubbe 1997: 33; cfr. anche Moraux 1960, il quale cerca di fare una netta distinzione tra le defixiones e le imprecazioni funerarie.

${ }^{17}$ Conseguentemente, ai fini della presente ricerca, sono stati inclusi tra le maledizioni, e quindi trattati, esclusivamente i testi epigrafici rispondenti a tale definizione.

18 Strubbe 1997: 33; va tenuto presente che questa distinzione viene definita dall'autore come 'artificiale'.

${ }^{19}$ Maledizioni 'greche' secondo la distinzione in Latte 1920: 77-78.

20 Maledizioni 'anatoliche' secondo la distinzione in Latte 1920: 78.
} 
proprietario del sepolcro ${ }^{21}$ e possiede un effetto immediato; questo tipo di maledizione, dunque, non implica necessariamente un intervento divino, ma agisce direttamente, attraverso la forza della parola iscritta, il Zauberkraft des ausgesprochenes Wortes, ${ }^{22}$ il cui potere deriva anche dal fatto che sia un morto a pronunciarla. ${ }^{23}$ Le imprecazioni del secondo gruppo, invece, contengono invocazioni agli dei, ${ }^{24}$ così che il proprietario della tomba conferisce la scelta del castigo alle divinità sotto la cui tutela è stato posto il sepolcro.

In base alle premesse appena fatte, è possibile individuare i due gruppi summenzionati anche tre le maledizioni attestate nelle province balcaniche.

Al primo gruppo, quello contenente minacce concrete esplicitanti i mali che ricadranno sul violatore, appartiene l'iscrizione classificata come Perinthos 180, proveniente da Perinto e databile al IV secolo d.C. ${ }^{25}$ In essa sono presenti

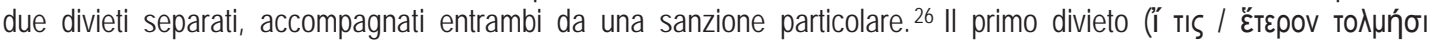

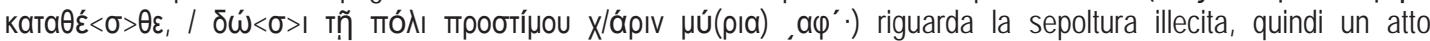
perseguibile dalla legge, che comporta una sanzione giuridica, ovvero il pagamento di una multa. II secondo divieto ( $\varepsilon \dot{~} \delta \varepsilon$

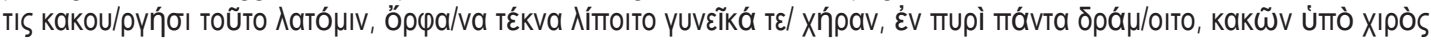

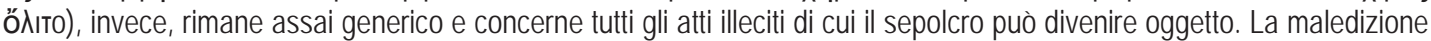
si esprime per mezzo di una formula prolissa e descrittiva, composta da quattro topoi interconnessi che alludono indirettamente alla morte del trasgressore - i figli orfani, la moglie vedova, la distruzione degli averi dal fuoco e la morte per mano di malintenzionati. Suddetti topoi, fatta eccezione per l'epigrafe trace qui analizzata, appaiono circoscritti all'ambito microasiatico. ${ }^{27}$ || topos degli őp $\varphi$ ava TÉkva $^{28}$ si riscontra una trentina di volte in Galatia, ${ }^{29}$ Phrygia, ${ }^{30}$ Lycaonia $^{31}$ e Pisidia ${ }^{32}$ e in quasi tutti i casi attestati, esso fa parte di una formula fissa e viene abbinato ad altri due

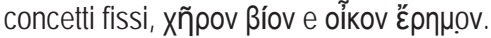

II topos della үuvعĩka Xńpav, ${ }^{33}$ invece, stranamente non è spesso abbinato al tema dei figli orfani, ma risulta scarsamente attestato e finora ne sono noti solo due casi, uno di Phrygia ${ }^{34}$ e uno di Lycaonia. ${ }^{35}$ È da notare la somiglianza di forma, se non anche di significato, tra i topoi della moglie vedova, xńpa, e la vita vuota, xñpos, di cui il secondo non solo è maggiormente diffuso, ma viene di regola abbinato al concetto dei figli orfani, con cui compone una formula fissa. È forse possibile, dunque, che le poche epigrafi che menzionino la үuveĩka xńpav siano frutto di

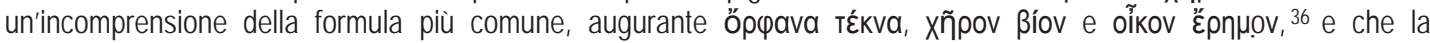
somiglianza fonetica (e anche etimologica) tra xńpa e хñpos abbia portato alla sostituzione del topos della moglie vedova a quello della vita vuota. ${ }^{37}$

Anche il terzo topos, quello della distruzione degli averi38 per mezzo di fuoco, è attestato raramente. ${ }^{39}$ Finora sono

\footnotetext{
${ }^{21}$ Strubbe 1997: 36 .

22 Latte 1920: 77-78.

${ }^{23}$ cfr. Strubbe 1997: 40; Valois 1914: 254-255.

24 Strubbe 1997: 36.

${ }_{25}^{25}$ Perinthos 180.

${ }^{26}$ In questo caso sarebbe possibile pensare a un legame tra il tipo di azione vietata e il tipo di sanzione conseguente, vale a dire che le azioni proibite dalla legge vengono punite con sanzioni giuridiche, mentre per le altre si usano le maledizioni. Tuttavia, nelle altre epigrafi analizzate finora mancano prove in favore a tale ipotesi.

${ }^{27}$ Per la differenza della concezione della sfera post mortem nell'Asia Minore e nella Grecia continentale, cfr. Strubbe 1997: 40 e Lattimore 1942: 108-117.

28 Tra le epigrafi in lingua latina il topos dei figli orfani che serve a alludere alla morte del violatore sembra sconosciuto.

${ }^{29}$ MAMA 1 294, 332; MAMA 7 214, 231, 254, 448, 535; JHS 19 (1899) 131,157; JHS 19 (1899) 289,189 JHS 19 (1899) 305, 244.

30 CIG 3862(2); MAMA 4 20, 85a; MAMA 7147, 266; MAMA 10 279; Haspels, Highlands of Phrygia 303, 20; Sterrett, EJ 171,164

Sterrett, WE 357,513/514; Waelkens, Türsteine 441, 510.

${ }^{31}$ CIG 3990k, 4000, 4000 [2]; MAMA 1 126, 235; MAMA 7 23, 28; RPh 1912:64,28; SEG 6:417.

32 Sterrett, EJ 158,144 Pisidia.

${ }_{33}$ Tra le epigrafi in lingua latina il topos della moglie vedova, alludente alla morte del trasgressore, sembra sconosciuto.

${ }^{34}$ MAMA 420.

35 MAMA 1235.

${ }^{36}$ La resistenza della formula potrebbe essere dovuta al fatto che si tratti di un esametro dattilico scorretto, cfr. Strubbe 1997: 42.

${ }^{37}$ Per la possibile interpretazione di xñpov Bíov come "la vita della vedova, vuota (priva) di marito", cfr. Robert 1978, 259-262; essa è tuttavia messa in dubbio da Strubbe 1997: 54, n. 87.

${ }_{38}$ Per la distruzione della proprietà materiale del violatore, cfr. Strubbe 1997: 43.

${ }^{39}$ Non sono noti paralleli latini che usano il topos del fuoco per indicare la distruzione del violatore e della sua proprietà.
} 
note solo sei epigrafi della Phrygia, ${ }^{40}$ della Lycaonia ${ }^{41}$ e della Pisidia ${ }^{42}$ in cui è presente la formula $̇$ mupì mávta

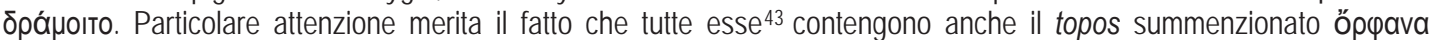

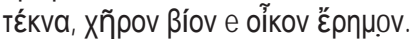

L'indagine condotta sulla diffusione del quarto topos, la morte per mano di malintenzionati, ha dimostrato che, a parte l'iscrizione di Perinto, esso risulta attestato solamente nelle stesse cinque epigrafi microasiatiche appena citate.

È possibile dunque concludere che si è in presenza di una formula particolare, caratteristica dell'Asia Minore, la

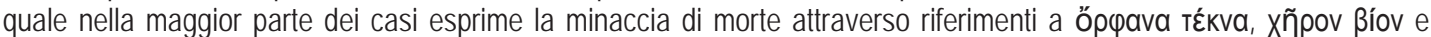

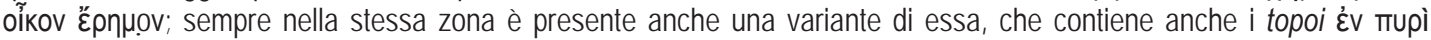

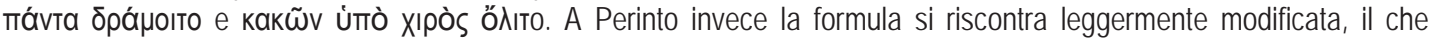
potrebbe essere dovuto sia a una semplice innovazione, sia a un'incomprensione dei topoi originali.

Una maledizione volta a provocare la morte del violatore del sepolcro è presente in un altro testo proveniente dalla Thracia, di Philippopolis (III d.C.). ${ }^{44}$ Anche in esso ritroviamo un duplice divieto (di aprire la tomba e di seppellirvi senza

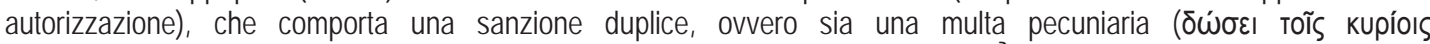

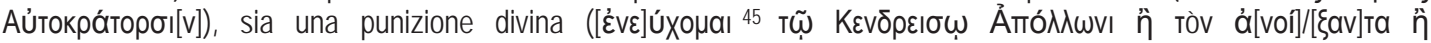

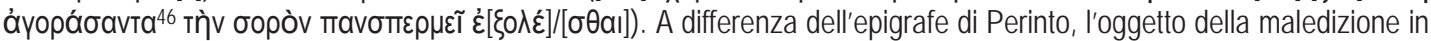
questione è direttamente il trasgressore dei divieti esposti, ma l'azione dell'imprecazione viene estesa attraverso il

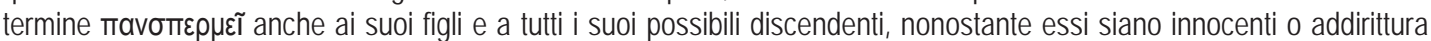
non ancora nati. ${ }^{47}$

Nell'epigrafe qui analizzata la maledizione viene espressa direttamente, attraverso il verbo exollymi. ${ }^{48} \mathrm{~A}$ prima vista la scelta della punizione, fatta a priori dal titolare della tomba, indurrebbe a includere l'imprecazione nel gruppo delle maledizioni non concrete che agiscono attraverso la forza magica della parola, non ricorrendo all'intervento divino. Tuttavia, l'invocazione ad Apollo Kendreisios, il quale dovrà provvedere alla realizzazione del castigo, dimostra chiaramente che si tratta di una maledizione 'mista' che contemporaneamente assegna alla potenza divina la facoltà di punire il trasgressore, ma non lascia alla dio libertà di agire, definendo chiaramente la punizione desiderata. ${ }^{49}$

L'invocazione ad Apollo Kendreisios merita particolare attenzione anche per una seconda ragione. Si tratta infatti di un culto locale, caratteristico esclusivamente della zona di Philippopolis. Tuttavia, il titolare del sepolcro (e quindi

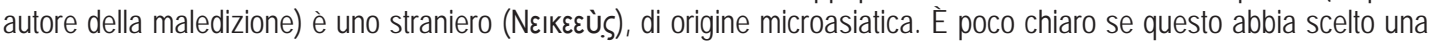
divinità locale, estranea alla sua religione originaria, come garante dell'incolumità della tomba in quanto lui stesso è diventato devoto a questo culto, o invece allo scopo di esercitare un'influenza maggiore sui violatori di tomba locali, fedeli di Apollo Kendreisios. Rimane dunque aperta la domanda se la scelta della divinità a cui rivolgersi per tutelare la tomba sia determinata dalla religiosità del defunto 0 dalle credenze dei destinatari della comunicazione epigrafica. ${ }^{50}$

Nell'ambito del gruppo delle minacce non concrete, invece, è possibile annoverare un'iscrizione coeva, proveniente sempre da Philippopolis. ${ }^{51}$ Essa riguarda di nuovo la sepoltura illecita e contiene una maledizione, espressa attraverso la formula semplice $\dot{\varepsilon} \xi o p \kappa i<\zeta \omega>\theta \varepsilon o u ̀ s$ kataxӨovíous, in cui la natura e il grado della punizione vengono lasciati alla scelta degli dei invocati. In questo caso l'invocazione è diretta non ad una divinità specifica, ma al gruppo generico degli dei inferi; si osserva dunque la pratica comunissima nel mondo antico di conferire la tutela del sepolcro alle divinità dell'oltretomba. Infatti, poiché la tomba appartiene al regno dell'aldilà, la violazione non rappresenta solo un

\footnotetext{
40 Sterrett, EJ 171,164; Waelkens, Türsteine 510.

${ }^{41}$ CIG 4000; CIG 4000 [2]; SEG 28:1249.

42 Sterrett, EJ 158,144P.

${ }^{43}$ A parte SEG 28:1249, la quale però è molto frammentaria.

44 IGBR III 1998.

45 Per l'uso del verbo euchomai e le maledizioni che hanno la forma di preghiera, cfr. Strubbe 1997: 45.

46 Il participio áyopáoavia dimostra che l'epigrafe si riferisce all'alienazione illecita del sepolcro e alla conseguente sepoltura illecita dei nuovi proprietari.

${ }^{47}$ cfr. Strubbe 1997: 43

48 L'augurio di morte, espresso in modo diretto attraverso vari verbi (apollymi, exollymi, perire, ecc.) è assai comune sia nelle epigrafi greche che in quelle latine ed è dunque impossibile elencare qui tutti i paralleli.

${ }^{49} \mathrm{Per}$ le maledizioni 'miste' cfr. Strubbe 1997: 35ss.

50 Per la scelta del dio a cui rivolgere le imprecazioni, cfr. Strubbe 1997: 46. Tuttavia, non bisogna dimenticare che la divinità locale viene concepita come 'proprietaria' o patrona del territorio in cui viene venerata, per cui potrebbe essere normale rivolgersi alla forza divina del luogo per sancire una garanzia di rispetto.

51 IGBR III 1999.
} 
crimine contro le leggi umane e contro i diritti privati del defunto, ma anche un'offesa alle divinità del mondo del postmortem, e dunque diviene quasi loro dovere punire i trasgressori. ${ }^{2}$ L'usanza di affidare il castigo del violatore alle divinità infernali impiega sovente il verbo $\dot{\xi} \xi o \rho \kappa i ́ \zeta \omega^{53}$ e si presenta con un funzione precauzionale, volta appunto a prevenire eventuali danni al sepolcro.

Assai simile all'epigrafe philippopolitana è un testo molto frammentario di Novae, ${ }^{54}$ di cui è conservata solamente la formula habebit deos iratos et Genium imp[- - -]. Anche qui è presente un'imprecazione non specifica, la quale menziona l'ira divina senza definire una punizione concreta, lasciando dunque la scelta agli dei invocati. Si tratta di un topos comune sia nelle epigrafi di lingua greca ${ }^{55}$ che in quelle latine,$^{56}$ il quale viene spesso abbinato a invocazioni a divinità concrete 0 , più in generale, agli dei celesti e/o inferi. Nel testo di Novae invece l'imprecazione è rivolta a un deos assai generico, equiparabile al greco pantes theoi o semplicemente theoi, ${ }^{57}$ alludente a tutte le divinità possibili. È molto probabile che la scelta di invocare non un dio specifico, ma l'intero panteon intenda moltiplicare la forza della maledizione stessa, in quanto, essendo la tomba posta sotto la tutela di più potenze divine, saranno tutte le divinità a dover punire i malfattori. ${ }^{58}$

Da notare è anche il secondo elemento dell'imprecazione: viene invocata l'ira anche di un Genium imp[- - -]. La tentazione di integrarvi Genium imp[eratoris] è assai forte; tuttavia finora non si conoscono iscrizioni in cui il genio dell'imperatore venga collegato alla sfera del post-mortem. Inoltre l'unica menzione di un Genius in contesto funerario si riscontra in CIL VI 29944, ove è presente un Genius populi Romani. Vista la mancanza di paralleli certi, il problema dell'interpretazione del Genius invocato nell'epigrafe di Novae rimane aperto.

Al tipo delle maledizioni non concrete che ripongono nelle divinità la speranza di una giusta punizione per i violatori appartiene anche il gruppo più ampio di maledizioni sepolcrali presente nelle province balcaniche, databili tra il IV e VI secolo d.C. e riunite da una formula in comune: invocazione diretta al Dio cristiano. In cinque delle epigrafi, 59 provenienti dalla Macedonia, è presente la frase $\delta \omega ́ \sigma \varepsilon ı ~ \lambda o ́ y o v ~ т \tilde{~} \theta \varepsilon \tilde{\omega}^{60}$ - si riscontra un parallelismo con il passo biblico äpa [oưv]

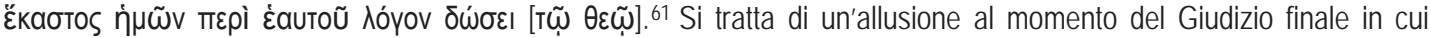
ognuno dovrà rendere conto delle sue azioni; è presente anche un accenno al peccato che potrebbe essere commesso violando il sepolcro e al conseguente castigo che verrà inflitto dalla giustizia divina. Lo stesso concetto si rileva

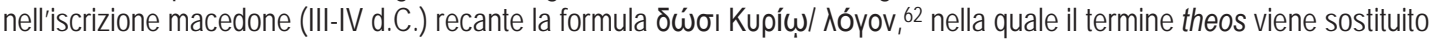

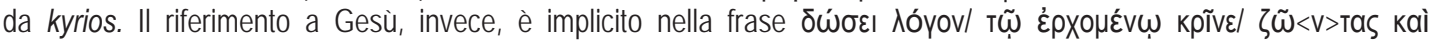
vعкрoús, ${ }^{63}$ attestata in Thracia; la menzione del Giudizio dei vivi e dei morti, tuttavia, rende la minaccia abbastanza

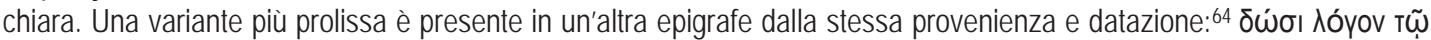

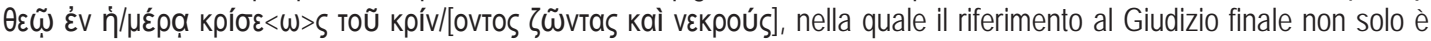
diventato esplicito, ma è anche appositamente potenziato per mezzo della ripetizione dei derivati del verbo krino -

${ }^{52}$ Cfr., per le multe destinate al fisco che coinvolgono le istituzioni civili, Libitina 2004: 391ss.

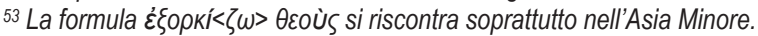

54 IGLNovae 108.

${ }^{55}$ cfr. Strubbe 1997: 45 e IG II' 13213; SIA VI 127; IG IX,2 1201; IC I XVIII 64; TAM V,1 423; TAM V,2 1371; Herrmann-Polatkan, Testament 7,1; Alt.v.Hierapolis 339; MAMA 6 List 150,186; MAMA 8 353; Sterrett, EJ 216,237; JHS 22 (1902) 346,82; MAMA 3 77; JHS 18 (1898) 307,3; MDAl(A) 27 (1902) 138,170.

${ }^{56}$ CIL 4, 538; 7716; 10486b; CIL 5, 3034; CIL 6, 1199; 13740; 19656; 21129; 25605; 29848b; 29944; 36537. 36664,08; 37530; 41434; CIL 8, 11825; 15716; 18261; CIL 10, 178; 2289; 2487; 4477; CIL 14, 01872; GraffPalatino 2, 268; Hep 13, 432; AE 1929, 228 ; $A E$ 2005, 1187; AE 2007, 148; TitAq 1, 159; 220; AE 1949, 48; AE 1974, 438; AE 1967, 42.

${ }^{57}$ Cfr. Strubbe 1997: 46

58 Un meccanismo simile si riscontra nei casi di multe sepolcrali, destinate a più istituzioni allo scopo di assicurare una tutela molteplice della tomba. Cfr. Libitina 2004: 391ss.

${ }^{59}$ RIChrM 32; 218; 219; 238.

${ }^{60}$ Riscontrabile anche in IG II² 13223 Att; Corinth 8,3 636I; 638; IG IX,1 643; IK Iznik 552; 555; 556; 557; 559; 573; St.Pont. III 30[2]; Strubbe, Cat. Pessinus 120; 122; 125; Haspels, Highlands of Phrygia 332,91; IK Pessinous 69; 85; 14; Waelkens, Türsteine 772; MAMA 1159; 163; 164; 164a; 167; MAMA 7 66; 93; JHS 22 (1902) 354,98; SEG 6:416; CIG 4441; finora non sono noti parallelismi con il summenzionato passo biblico tra le epigrafi in lingua latina. Per l'interpretazione della formula cfr. Robert 1960: 407.

${ }^{61}$ Rom. 14. 12.

62 RIChrM 58, Macedonia.

${ }^{63}$ Perinthos 185 e 199 (lett. risponderà a colui che verrà a giudicare i vivi e i morti).

${ }_{64}$ Perinthos 186, III secolo d.C. 


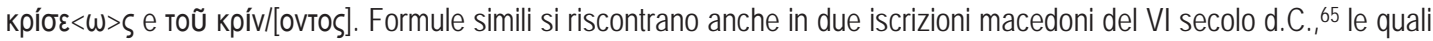

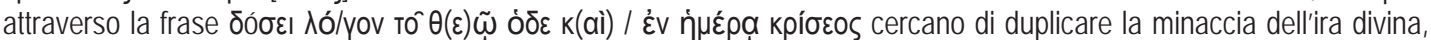
collocandola contemporaneamente nel momento della violazione e nel giorno del Giudizio. 66

È possibile, infine, che un'ultima epigrafe macedone del IV secolo d.C. ${ }^{67}$ vada riferita allo stesso gruppo di imprecazioni, nonostante non contenga la formula $\delta \omega ́ \sigma \varepsilon ı ~ \lambda o ́ y o v ~ т \tilde{~} \theta \varepsilon \tilde{\omega}$. Si tratta di un'iscrizione cristiana, la quale

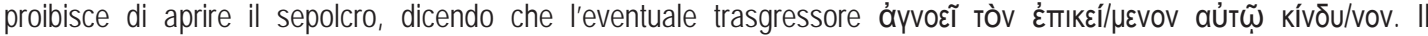
riferimento a un pericolo imminente, di cui il violatore non è cosciente, è poco chiaro; visto il contesto cristiano, tuttavia, sarebbe possibile pensare che il testo richiami appunto il concetto del Giudizio finale, durante il quale i peccati, tra cui anche la violazione di sepolcri, verranno puniti dalla giustizia divina. Se così fosse, la formula in questione

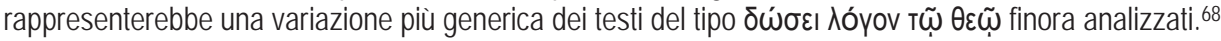

\section{Conclusioni. Nuovi contenuti spirituali del mondo tardoantico}

Dall'indagine condotta nel materiale epigrafico esaminato sembra delinearsi una situazione sensibilmente differente da quanto finora emerso dagli studi precedenti, di cui si è fatto cenno nell'Introduzione, che sottolineano usualmente l'impossibilità di tratteggiare uno sviluppo diacronico dell'affermazione e diffusione della maledizione sepolcrale in Oriente, a causa dell'eterogeneità dei dati disponibili.

Dall'analisi sistematica delle iscrizioni con maledizioni emerge, al contrario, un chiaro paradigma evolutivo del fenomeno, che si inizia a manifestare in maniera consistente, per quanto non ancora segnatamente diffusa, nella prima età imperiale. Le formule impiegate in quest'epoca trovano riscontro per lo più in ambiente orientale, mostrando infatti una chiara derivazione di matrice microasiatica, come si evince dai paralleli precedentemente riportati.

L'impiego della maledizione sepolcrale nell'area considerata conosce un deciso aumento - contestuale all'inasprirsi della legislazione pertinente la violatio sepulcri - a partire dalla fine del II secolo-inizio III secolo e una stabile diffusione durante i secoli IV, V e VI d.C. Tale processo appare sintomatico della trasformazione verso la Tarda Antichità in atto, che registra in primo luogo il cambiamento degli atteggiamenti mentali e dei contenuti spirituali.

La lenta trasformazione delle strutture mentali e religiose nel periodo tardoantico si legge, infatti, nel sempre più frequente - e infine esclusivo - ricorso all'invocazione del Dio unico ma anche nell'aumento stesso dell'attenzione per la tomba, nonché nella credenza dell'efficacia del mezzo religioso quale strumento per la sua preservazione - caratteri che esprimono, difatti, la trasformazione dei contenuti culturali del mondo tardoantico verso una maggior attenzione per la vita religiosa e specificatamente per l'esistenza post-mortem. ${ }^{69}$

Le iscrizioni sottolineano quindi la trasformazione in senso cristiano del mondo antico, in particolare balcanico, che non appare affatto segnato né da declino né da crisi, né dallo 'scetticismo dilagante' rilevato dal Parrot. La fase compresa tra III e VI d.C. non si configura neppure come un momento di transizione, quanto piuttosto un'epoca dotata di piena autonomia che vede la trasformazione dei valori classici in nuovi dati e si accompagna, parimenti, a un cambiamento che investe anche le strutture sociali ed economico-produttive.

In maniera particolare, le iscrizioni esaminate, per la natura del loro contenuto e per il loro incrementato numero, mostrano l'importanza che la morte ha acquisito nel mondo tardoantico, avendo il Cristianesimo assegnato un posto centrale al tema della fine della vita umana e dell'immortalità. ${ }^{70}$ La nuova religione, realizzando un rapporto tra la cultura classica e le altre culture, spesso di matrice orientale, con cui questa era entrata in rapporto, raccoglie la tradizione classica, acquisendola, inglobandola e trasformandola: in ultima analisi, il processo di mutamento interno che conduce il

\footnotetext{
65 SEG 41:572,B e C.

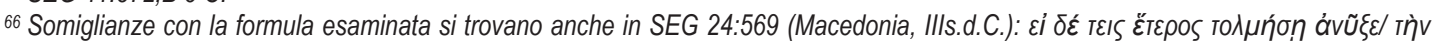

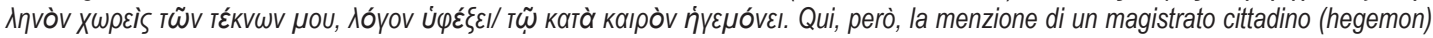
porta a includere il testo nel gruppo delle sanzioni giuridiche.

${ }_{67}$ RIChrM 123.

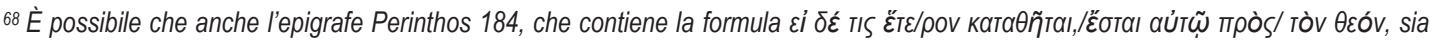
da attribuire a questo tipo.

${ }^{69}$ L'invecchiamento' della società tardoantica è correlato da Burkhardt ai nuovi contenuti culturali determinati dal Cristianesimo, fattore decisivo della trasformazione dell'epoca, come debitamente sottolineato da J.H. Renàn (1877), ma anche da E.R. Dodds (1991) il quale identifica nel Cristianesimo una componente essenziale del cambiamento strutturale dell'età imperiale verso il mondo tardoantico.

${ }^{70}$ L'opera The Conversion of Constantine and Pagan Rome di G. Alföldy mette in rilievo il passaggio, in questa fase, dalla cultura antica verso forme di religiosità diverse, non più poliadiche, ma legate all'immortalità.
} 
mondo tardoantico a convergere verso il Cristianesimo non rappresenta una lotta tra istanze differenti, ma costituisce il naturale risolversi di un processo in cui una cultura - quella classica - che ha perso le sue caratteristiche confluisce in un'altra, che ne è allo stesso tempo la continuazione.

Se le maledizioni funerarie possono essere lette come uno strumento in cui appaiono riflesse le credenze fondanti di una civiltà, quelle attestate in ambito balcanico nei secoli III-VI, pur facendo proprie espressioni e strutture sintattiche ereditate della tradizione greco-romana, che in esse confluiscono e in alcuni casi permangono, implicitamente enucleano i nuovi caratteri spirituali del mondo tardoantico: la centralità acquisita dalla morte e dalla vita ultraterrena, da cui scaturisce la particolare preoccupazione per la tomba e la sua tutela; la correlata credenza nell'immortalità; il venir meno della religione pagana e delle divinità del pantheon greco-romano; l'affermarsi del Cristianesimo e del culto del Dio unico; il diffondersi della credenza nel giorno del giudizio, nel concetto del peccato, di cui il reo dovrà rispondere.

Inoltre, pur non essendo questo l'oggetto di interesse del presente studio, le iscrizioni funerarie contenenti maledizioni consentono di cogliere, attraverso la menzione di pene finanziarie, anche altri aspetti, in particolare socioeconomici del mondo tardoantico, quali la persistenza di un'economia a base monetale o il lento affermarsi della Chiesa quale destinataria delle multe riscosse.

\section{Bibliografia}

Cumont 1930: Fr. Cumont, Un rescrit impérial sur la violation de sépulture, "Revue historique" 163, 1930, $241-255$.

Cuq 1932: E. Cuq, Les violations de sepultures, "Revue historique de droit français et étranger" 1932, 109-126.

De Visscher 1953: F. De Visscher, Les peines sepulcrales, in Festschrift für H. Lewald, Basel 1953, 175-183.

De Visscher 1963: F. De Visscher, Le droit des tombeaux romains, Milano 1963.

Dodds 1991: E.R. Dodds, Pagans and Christians in the Age of Anxiety: Some Aspects of Religious Experience from Marcus Aurelius to Constantine, Cambridge 1991.

Giorgi 1910: G. Giorgi, Le multe sepolcrali nel diritto romano, Bologna 1910.

Huschke 1874: Ph. Huschke, Die Multa und das Sacramentum in ihren verschiedene Anwendungen, Leipzig, 1874.

Latte 1920: K. Latte, Heiliges Recht: Untersuchungen zur Geschichte der sakralen Rechtsformen in Griechenland, Tuebingen 1920.

Lattimore 1942: R. Lattimore, Themes in Greek and Latin Epitaphs, "Urbana" 3, 1942, 108-117.

Lazzarini 1991: S. Lazzarini, Sepulcra familiaria. Un'indagine epigrafico-giuridica, Padova 1991.

Lecrivain 1904: Ch. Lecrivain, Multa, in Mm. Ch. Daremberg - Edm. Saglio (éds.) Dictionnaire des Antiquités Grecques et Romaines, III, 2, Paris 1904, 2014-2020.

Libitina 2004: AA.VV. Libitina e dintorni. Libitina e i luci sepolcrali; le Leges Libitinarie campane; lura sepulcrorum. Vecchie e nuove iscrizioni, Roma 2004.

Mommsen 1907: Th. Mommsen, Zum roemischen Grabrecht, in Gesammelte Schriften, III, Juridische Schriften, Berlin 1907, $198-214$.

Moraux 1960: P. Moraux, Une defixion judiciaire au Musee d'Istanbul, "Mémoires de l'Académie royale des sciences, des lettres et des beaux-arts de Belgique" 54, 2, 1960.

Parrot 1939: A. Parrot, Maledictions et Violations de Tombes, Paris 1939.

Renàn 1877: J.H. Renàn, Histoire des origines du Christianisme, Paris 1877.

Ritti 2004: T. Ritti, Iura sepulcrorum a Hierapolis di Frigia nel quadro dell'epigrafia sepolcrale microasiatica. Iscrizioni edite e inedite, in AA. VV. Libitina e dintorni. Libitina e i luci sepolcrali; le Leges Libitinarie campane; lura sepulcrorum. Vecchie e nuove iscrizioni, Roma 2004, 455-634.

Robert 1960: L. Robert, Hellenica, XI-XII, Paris 1960.

Robert 1978: L. Robert, Maledictions funeraires grecques, "Comptes-rendus des séances de l'Académie des Inscriptions et BellesLettres", 1978.

Sottas 1910: H. Sottas, La préservation de la propriéte funérarie dans l'ancienne Egypte, Paris 1913.

Sterrett 1988 = E.J. Sterrett, An Epigraphical Journey in Asia Minor during the summer of 1884, «Papers of The American School of Classical Studies at Athens» 2, 1883/84, Boston 1888.

Strubbe 1991: J.H.M. Strubbe, Cursed be he that moves my bones, in C.A. Faraone - D. Obbink (Eds.), Magika Hiera. Ancient Greek Magic and Religion, New York-Oxford 1991.

Strubbe 1997: J.H.M. Strubbe (Ed.), APAI EPITYMBIOI. Imprecations against Desecrators of the Grave in the Greek Epitaphs of Asia Minor. A Catalogue, Bonn 1997.

Tosi 1993: M. Tosi, Multae, comminationes, dirae nelle iscrizioni funerarie transpadane, pagane e cristiane, "Rivista archeologica della Città e Provincia di Como" 175, 1993, 189-240.

Toynbee 1996: J.M.C. Toynbee, Death and Burial in the Roman World, New York 1996.

Tsalampouni 2001: E. Tsalampouni, The Nazareth inscription. A controversial piece of palestinian epigraphy (1930-1999), "Tekmēria: Contributions to the History of the Greek and Roman World" 6, 2001, 70-120.

Valois 1914: R. Valois, APAl, "Bulletin de Correspondance Hellénique" 38, 1914, 250-271. 


\section{Abbreviazioni}

$\mathrm{AE}=$ Archaiologike Ephemeris

$\mathrm{CIL}=$ Corpus Inscriptionum Latinarum

$\mathrm{CIG}=$ Corpus Inscriptionum Graecarum

IC = Inscriptiones Creticae

IG = Inscriptiones Graecae

IGLNovae = Inscriptions grecques et latines de Novae (Mésie inférieure)

IGBR = Inscriptiones Graecae in Bulgaria repertae

IK Pessinous = J. Strubbe, The Inscriptions of Pessinous, «Inschriften griechischer Städte aus Kleinasien» 66, Bonn 2005

JHS = The Journal of Hellenic Studies

MAMA = Monumenta Asiae Minoris Antiqua

$\operatorname{MDAI}(A)=$ Mitteilungen des deutschen archäologischen Instituts. Athenische Abteilung

RIChrM = D. Feissel, Recueil des inscriptions chrétiennes de Macédoine du Ille au Vle siècle, «Bulletin de correspondance hellénique. Supplément», 8. Paris 1983

$\mathrm{RPh}=$ Revue de philologie, de littérature et d'histoire anciennes

SEG = Supplementum Epigraphicum Graecum

$\mathrm{SIA}=$ Supplementum Inscriptionum Atticarum

TAM = Tituli Asiae Minoris

TitAq = Tituli Aquincenses 